\title{
The Rise and Decline of Arabu-Tamil Language for Tamil Muslims
}

\author{
Dr. K. M. A. Ahamed Zubair*
}

\begin{abstract}
The Arab Muslim Traders and the native Tamil converts to Islam in Tamilnadu state of India and Sri Lanka came into closer contact as a result of their commercial activities. They were bound by a common religion, but separated by two different languages They felt the necessity for a link-language. They started to write Tamil in an adapted Arabic script called Arabu-Tamil. The Arabu-Tamil or Arwi script represents the Tamil language using an Arabic style of scripts. From eighth century to nineteenth century, this language enjoyed its popularity among Tamil speaking Muslims of Tamil Nadu and Sri Lanka. The valuable and useful ideas of Tamil Muslim minds were conveyed in Arabicized Tamil called Arabu-Tamil. It rendered a most useful service for the advancement and progress of Arab and Tamil cultures. However, the beginning of the twentieth century saw the decline of Arwi language. And no step was taken to arrest this decline. The study analyses its rise and decline as a language of Tamil Muslims.
\end{abstract}

\section{Introduction}

Arwi or Arabu-Tamil is an Arabic-influenced dialect of the Tamil language written with an extension of the Arabic alphabet, with extensive lexical and phonetic influences from the Arabic language. Arabu-Tamil was used extensively by the Muslim minority of Tamil

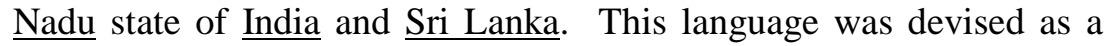
means for communication and interaction of the settled Arabs of Tamil Nadu to the Tamil people. It is an outcome of the cultural synthesis between seafaring Arabs and Tamil-speaking Muslims of Tamil Nadu.

\footnotetext{
*Assistant Professor of Arabic, The New College, Tamil Nadu, India.
} 
It was popular in Tamil Nadu and Ceylon. It has a rich body of literature of which little has been preserved. There are historical records of the prevalence of Arabu-Tamil in far Eastern countries, such as Indonesia and Thailand, up until the 1970s. ${ }^{1}$

Arabu -Tamil served as a medium of transformation of the Muslim Tamil society of Tamilnadu and Sri Lanka in their daily affairs to write many religious, literary and poetry texts for communication. The Arabu -Tamil script represents the Tamil language (having left-to-right script) using an Arabic style of script (having right-to-left script). The Arwi or Arabu-Tamil script was widely used by the Muslim Tamils of Asia for their day-to-day communication. From $8^{\text {th }}$ century to the $19^{\text {th }}$ century, this language enjoyed its popularity among Tamil speaking Muslims of Tamil Nadu and Ceylon. It continues to enjoy the same popularity with the Muslims of Ceylon even today. The Tamil speaking Muslims of Ceylon consider this Arabu-Tamil literature as their most beloved literature. ${ }^{2}$ However, after the $19^{\text {th }}$ century, it began to lose its popularity primarily because of its old style and colloquial expression. As a spoken language it is now about to be extinct, though a few Madrasas still teach the basics of the language as part of their curricula.

The primary objective of this study is to share the magnificent literary history and the Islamic legacy of Arabu -Tamil language and literature to the Islamic world. This paper makes an analysis of its rise and decline as a language of Tamil Muslims.

\section{Tamil and Muslims}

Tamil is a Dravidian language spoken predominantly by Tamil people of the Indian subcontinent. It has official status in the Indian state of Tamil Nadu. Tamil is also an official language of Sri Lanka and Singapore. Tamils are a linguistic and ethnic group native to Tamil $\underline{\mathrm{Nadu}}$, a state in India and the north-eastern region of Sri Lanka. Tamil Muslim refers to the Muslims who have Tamil as their mother tongue. Apart from India and Sri Lanka, a large population of Tamil Muslims also live in some countries like Malaysia, Singapore and Mauritius and also in Gulf countries like UAE and Saudi Arabia. Tamil is also spoken by these Tamil minorities in those countries.

\section{Islam in Tamil Nadu}

Long before the birth and rise of Islam, there were frequent contacts between Arabia and Tamil Nadu, and there were Arab Colonies in 
South India. The classical Tamil literature, like in the literary works like Paththu Pattu, Ettuthogai, bears evidence of the Arab contact with Tamil Nadu. After its birth, Islam came to Tamil Nadu and Sri Lanka through these contacts. After the rise of Islam, the Arabs continued to remain actively in contact not only as traders but also as missionaries. So Trade and Islam flourished actively and the Muslims were permitted to follow their religion freely. The companions of the Holy Prophet Mohammed (peace and blessings of Allah be upon him) namely Saint Tamim al-dari al-Ansari (peace be on him), Saint Ukkaza (peace be on him) were buried in Tamil Nadu.

Tamil Muslims of Tamil Nadu are famous figures and they contributed their best to the Tamil Culture and Civilization. The Rulers of these areas patronized Islam and Muslims to flourish in Tamil Nadu. Some of them even donated lands to places of Muslim worship (Masjid). They include the Kings of Chera, Chozha and Pandyas. Muslims built Mosjids in various places of this country. The first and foremost among such Mosjids is the one which can be seen even today near the Railwany Station in Tiruchirapalli in the Tamil Nadu State of India. However, it is now in a dilapidated condition. The Arabic inscription stone available in that Masjid indicates that it was constructed in the year 734 (corresponding $116 \mathrm{AH}$, Islamic Era), and it was built by Mohammed bin Hamid bin Abdullah. This proves that Muslims lived in this region in the $8^{\text {th }}$ century liberally with all proprietary rights in this land. ${ }^{3}$

\section{Islam in Sri Lanka}

The Muslims are the second largest minority in Sri Lanka approximating to eight percent of its population. In Sri Lanka, the Arab settlements were along the coast of Jaffna, Mannar, Kendremalai, Puttalam, Colombo, Beruwala, Galle and at the eastern port of Trincomalle. A large population of the Moor population in Sri Lanka is descended from the traders who arrived from Arabia and the lands around the Persian Gulf. ${ }^{4}$

The similarities of the socio-religious paradigms of the Muslims of South India and Sri Lanka are quite enormous. These may be summarized as follows ${ }^{5}$ :

a. The home language of Sri Lankan Muslims and those of Tamil Nadu is Tamil. 
b. The Muslim Tamil (spoken at home) in Sri Lanka and Tamil Nadu is a dialect in that it has substantial number of TurkoPerso-Arabic loan words

c. There are close cultural systems between the Muslims of Sri Lanka and Tamil Nadu

d. Muslim Missionaries in Tamil Nadu throughout the ages have entered Sri Lanka and helped shape the religious thinking of the Muslims of Sri Lanka. These Muslim missionaries helped in the establishment of Tariqa. Most of the Qadiriyya sub-fraternities in Sri Lanka originate from their headquarters in Kilakarai, Kayalpattanam and Kottaru. Kilakarai and Kayalpattanam are ports in Ramnad and Tirunelveli districts respectively. Kottaru is a town in Tamil Nadu, close to the Kerala state .

\section{The Rise of Arabu-Tamil}

\section{Origin}

Arabu-Tamil was used by Arabs who came and settled in Tamil Nadu. The settled Arabs in Tamil Nadu learnt Tamil through Arabic Script and wrote Tamil in Arabic script. It is believed that the origin of Arabu-Tamil is as old as contact of Arabs with Tamil Nadu. ${ }^{6}$ The Muslim communities of Sarandib (Sri Lanka ) and Tamil Nadu were able to use this language as an effective shield for the preservation of their cultural identity. They were able to safeguard their culture without sacrificing their religion. ${ }^{7}$ Arabu-Tamil was the product of the cultural synthesis between the Arabs and the Tamil speaking Muslims. It is a combination of Arabic and Tamil, but written in Arabic script. After the advent of Islam the Arabs who already settled down in the area naturally became the representatives of Islam in these areas. In course of time, there were also converts to Islam from among the native population. When two communities, bounded by a common religion but separated by two different languages, came into closer contacts, a result of their commercial activities, they felt the necessity for a link-language. This ultimately gave birth to the Arabu-Tamil language. Arabu Tamil represents the fusion of two great languages, belonging to the great ethnic groups, one being the Semitic-Arabic and the other the Dravidian-Tamil.

The Arabs of Tamil Nadu wanted to learn Tamil, which was also an ancient language like Arabic. They started learning Tamil with the 
help of their own Arabic script. Having learnt this, they started conveying their ideas in this newly originated and newly blended language. In early days, the Arabs were accustomed to colloquial style, when they adopted this they completely ignored Tamil Grammar. They used Tamil Language in daily life as the English men used it in later days. It must be noted that the Arabs before writing the Tamil in Arabic script, used to remember orally most of the words of daily use. Later they put the same in Arabic script. This way the Arabu-Tamil language was originated in Tamil Nadu. ${ }^{8}$ In the beginning, the Arabians conveyed their ideas in words and the Tamilians absorbed, grasped and adopted these words and used them in their daily life and these words were considered to be most important ones for commercial purposes. Even today most of these words are in use. They are used not only by the Tamilians but also by the people of India. Following are the words of Arabic which are in common use': Amul, Asal, Aajar, Kaidi, Kajana, Mahajar, Jamin, Diwan, Dawa, Jilla, Taluk, Nakal, Mile, Jabthi, Ameena, Masoda, Munsif, Wakkil, Inam, Sharathu, Mahsool, Wasool, Mirasa, Varisu, Layak, Baqi, Thayathu, Maramathu, Jawabu, Kammi, Sharbath, Kesari, Jubba, Faisal, Thakararu, Raseedu, Thakal, Pasali (Fasel), Maamul, Maji, Maidanam (Maidan), Vagaiyera...etc.

Again, Tamil words lack the capacity to convey the full significance of the Islamic terms like Rasul, Sahabah, Wajib, Jannath, Jahannam, Iman, Taqwa etc., (their respective meanings are Messenger, Companions of Holy prophet, Obligatory, Paradise, Hell, belief, Piety...etc). Therefore Arabu-Tamil words are used in these cases for better expression and understanding. Hence the Tamil Muslims naturally preferred the Arabu-Tamil Language. The new language was shaped in a manner that retains all the basic aspects of the finest spoken Tamil, and all of its grammatical forms, put on the beautiful garb of the Arabic script. Arabic letters with suitably added diacritical marks were devised. Arabu-Tamil still has a place among the more traditional Indian Tamil Muslim and Sri Lankan Moor families. Today, it exists only informally in the speech of the Tamil spoken Muslims, as many words unique to it are used in their spoken Tamil. Some of these words that constitute daily conversations among Muslims for example are: Museebah, Mowth, Janazah, Balaah, Raahat, Shifaa, Khair, Wallahi, Ta'lim, Kithaab, Shaitaan, Sharbath, Sahan, Dafs, Baith, Bayaah, Shirk, Tayyib and Ikhlaas. 


\section{Place of origin}

The fact that Arabu-Tamil was prevalent in Colombo, Kayalpattinam, Kilakarai indicates that it was in use, as early as the eighth century of the Christian era. ${ }^{10}$ The Arabs and the Tamil Muslim might have played their role equally in the formation of Arabu-Tamil. It is the logical result of joint efforts of the Arabs and the Tamil Muslims. It originated in South-West Coast of Ceylon as well as in the SouthEast Coast of India, more particularly in Kayalpattinam. This language was enriched, promoted and developed in Kayalpattinam. It rendered a most useful service for the advancement and progress of Arab culture and Tamil culture.

\section{Arabic, Tamil and Arabu-Tamil Scripts}

The Arabu-Tamil consists of 40 letters, out of which 28 letters are from Arabic and 12 letters are devised by adding some marks and dots to the original Arabic Alphabet. Eighteen Arabic letters do not have their equivalents in Tamil from the phonetic point of view and similarly ten Tamil letters and two vowel sounds have no equivalents in Arabic. For a make-up the 12 letters were devised. Thus the ArabuTamil alphabet is the Arabic alphabet with the devised twelve additional letters to represent the Tamil vowels and several Tamil consonants that could not be mapped to Arabic sounds.

Arabic follows the consonantal system i.e it has distinct symbols or letters only for consonants, while the vowels are optional and not represented by separate letters but by a few diacritical marks without which Arabic texts can still be readable and understood. Arabic has 28 consonants and it is written usually from right to left. Tamil has 30 basic letters comprising 12 vowels 18 consonants and follows essentially a syllabic system of writing, the combination of the consonant and the vowel is represented by a syllabic symbols or letters. Tamil has 216 syllabic symbols or letters apart from the basic symbols or letters of vowels and consonants and it is written from left to right. 
The Arabic script is as follows:

\begin{tabular}{|c|c|c|c|c|c|c|}
\hline$\dot{\tau}$ & $\tau$ & $\varepsilon$ & 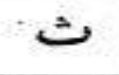 & $ت$ & 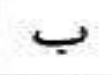 & i \\
\hline kha' & h'aa' & jiim & thaa' & $\operatorname{taa}^{3}$ & baa' & alif \\
\hline ص & ث & سى & j & 3 & j & 3 \\
\hline saad & shiin & $\sin$ & zaay & raa' & thaal & daal \\
\hline 3 & ت & $\dot{\varepsilon}$ & $\varepsilon$ & b & $b$ & ض \\
\hline qaaf & feh' & ghayn & 'ayn & thaa & Taa' & daad \\
\hline ي & 9 & • & 3 & i & $J$ & s) \\
\hline yaa' & waaw & haa & nuun & miim & laam & kaaf \\
\hline
\end{tabular}

In addition to Arabic script, 13 characters were added in Arwi script. Arwi is conveniently written with vowels. For those vowels which also exist in Arabic, that is -a-, -i-, -u-, -aa-, -ii-, -uu-, and the diphthongs ai- and -au-, the same signs are in use that are used in Arabic, i.e. fatha, qasra and damma for the short vowels, long vowel being indicated by additional alif, yaa and waaw, respectively, and the diphthongs being written with fatha (-a-) plus yaa or waaw. In addition to the basic Arabic letters, several characters have been introduced to write Tamil sounds not found in Arabic.

The Tamil script is as follows:

Tamil script of Vowels, Consonants and Syllabic Letters

\begin{tabular}{|c|c|c|c|c|c|c|c|c|c|c|c|c|}
\hline & श & ஆ & இ & F. & 2 & ஊ & எ & ஏ & ஐ & ஒ & ஓ & ஒள \\
\hline க் & க & கா & கி & கீ & கு & கூ & கெ & கே & கை & கொ & கோ & கௌ \\
\hline ங் & Ђ & ஙா & ஙி & ஈீீ & |ு। & நூ & ஙெ & ஙே & ஙை & ஙொ & ஙோ & Һெ \\
\hline$\dot{ச}$ & ச & சா & சி & சீ & சு & சூ & செ & சே & சை & சொ & சோ & செ \\
\hline ஞ் & 6 & & ஞி & ஞீ & ஞ & бூ & எெ & C, & & & & \\
\hline
\end{tabular}


IIUC Studies, Vol. 10 \& 11

Tamil script of Vowels, Consonants and Syllabic Letters

ட் ட டா டி டீ டு டூ டெ டே டை டொ டோ டௌ

ண்ணணாணி ணீ ணு ணெ ணேணை ணெ ணே ணெ6

த் த தா தி தீ து தூ தெ தே தை தொ தோ தௌ

ந் ந நா நி நீ நு நூ நெ நே நை நொ நோ நௌ

ப் ப பா பி பீ பு பூ பெ பே பை பொ போ பௌ

ம் ம மா மி மீ மு மூ மெ மே மை மொ மோ மௌ

ய் ய யா யி யீ யு யூ யெ யே யை யொ யோ யௌ

ர் ர ரா ரி ரீ ரு ரூ ரெ ரே ரை ரொ ரோ ரௌ

ல் ல லா லி லீ லு லூ லெ லே லை லொ லோ லௌ

வ் வ வா வி வீவு வூ வெ வே வை வொ வோ வெள

ழ் ழ ழா ழி ழீ ழு ழூ ழெ ழே ழை ழொ ழோ ழௌ

ள் ள ளா ளி ளீ ளு ளூ ளெ ளே ளை ளொ ளோ ளௌ

ற் ற றா றி றீ று றூ றெ றே றை றொ றோ றௌ

ன் ன னா னி னீ னு னூ னெ னே னை னொ னோ னௌ

$\therefore$ 
The Arabu-Tamil or Arwi script :

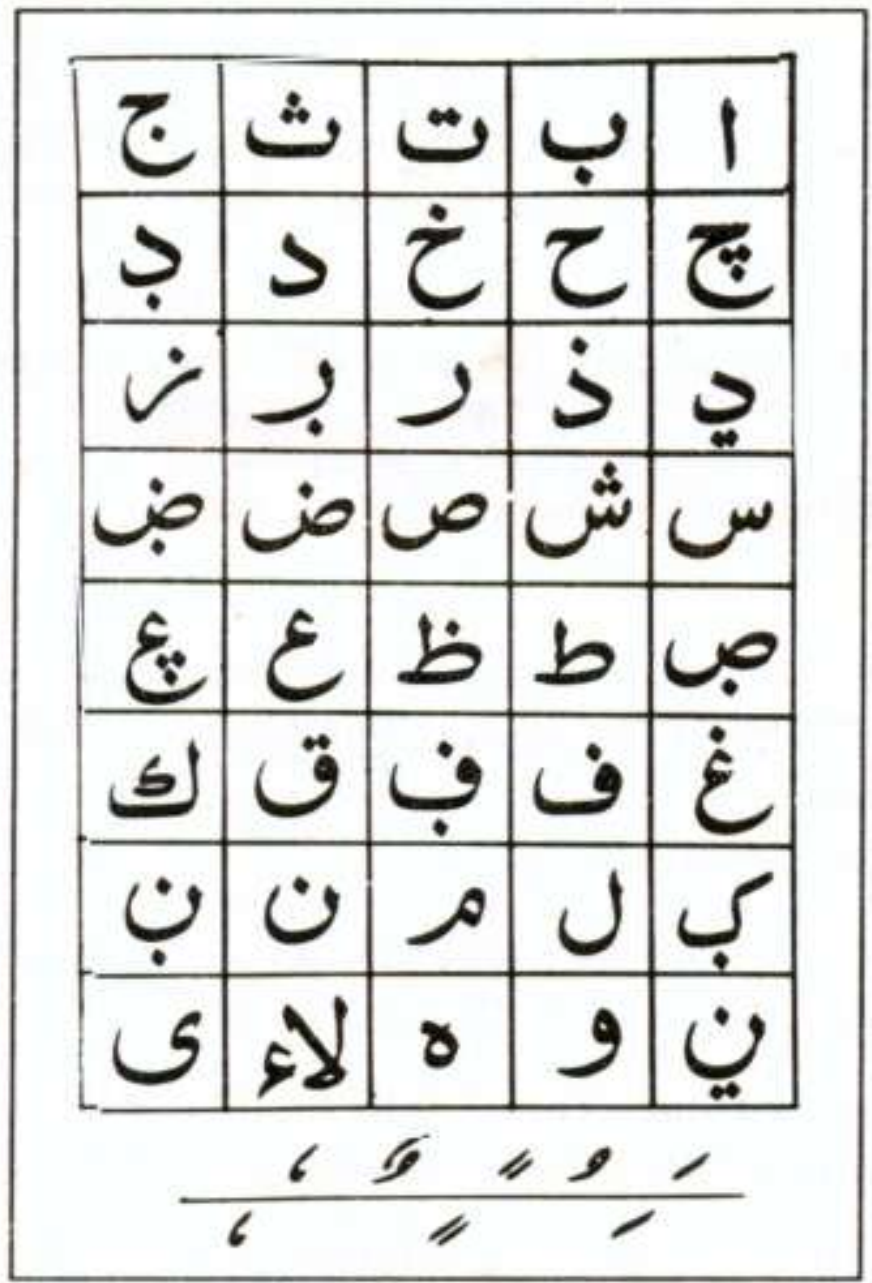


Specimen of Arabu-Tamil writing (Hand written) ---- 1

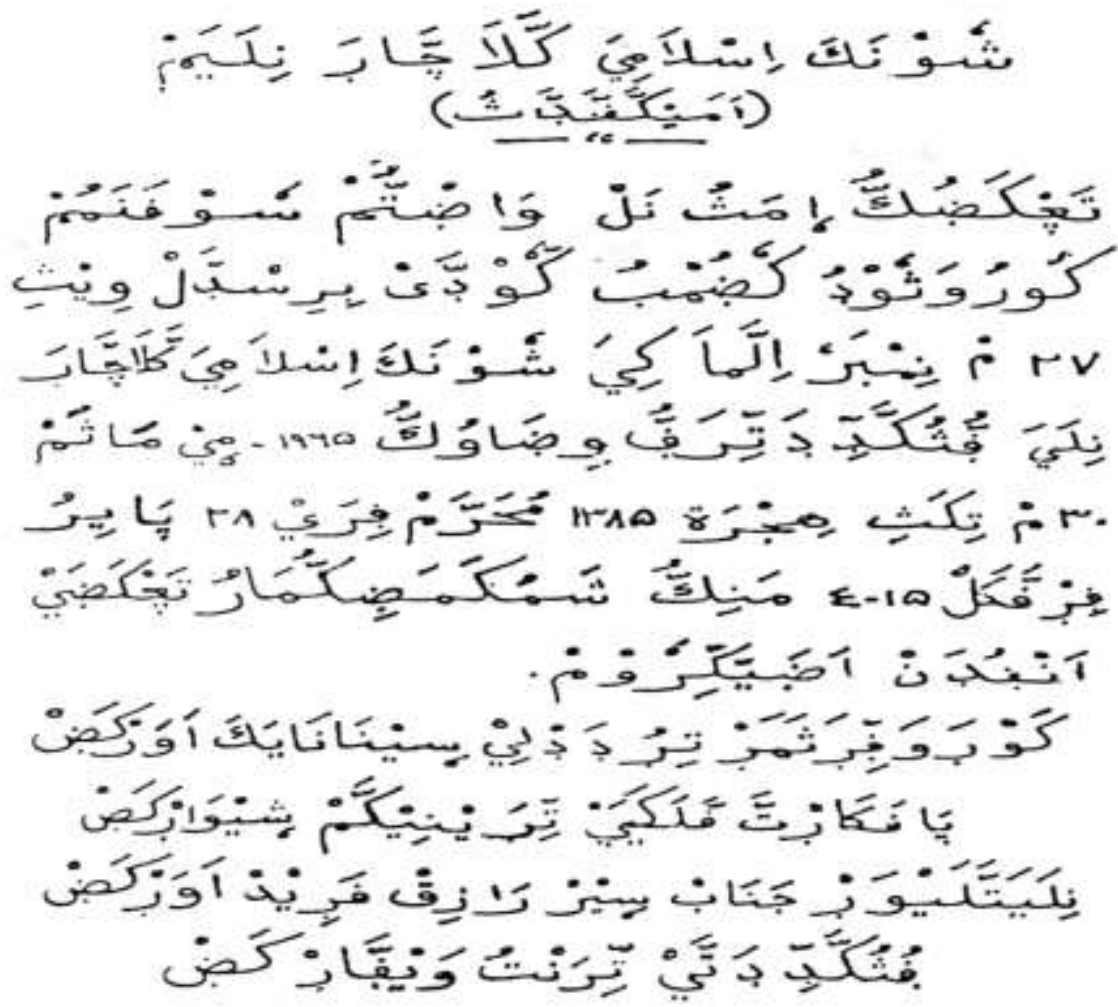

(Transliteration of the above Invitation in Tamil)

சோனக இஸ்லாமிய கலாச்சார நிலையம் (அமைக்கப்பட்டது)

தங்களுக்கு எமது நல்வாழ்த்தும் சோபனமும் கூறுவதோடு கொழும்பு கோட்டை பிரிஸ்டல் வீதி, 27 ஆம் நம்பர் இல்லமாகிய இஸ்லாமிய கலாச்சார நிலைய புதுக்கட்டிட திறப்பு விழாவிற்கு 1965, மே மாதம் 30 ஆம் தேதி, ஹிஜ்ரா 1385 முஹர்ரம் 28 பிறை 
ஞாயிறு பிற்பகல் 4:15 மணிக்கு சங்கமிக்குமாறு தங்களை அன்புடன் அழைக்கிறோம்.

கௌரவ பிரதமர் திரு.டட்லி சோனானாயக அவர்கள் ஞாபகார்த்த பலகையை திரை நீக்கம் செய்வார்கள். தலைமை நிலையத் தலைவர் ஜனாப் சர் ராஜிக் ஃபரீ த் அவர்கள் புதுக்கட்டிடத்தை திறந்து வைப்பார்கள்.

\section{(Translation of the above Invitation in English)}

\section{Sonaga ${ }^{11}$ Islamic Cultural Centre (Established)}

We send you our felicitations, greetings and cordially invite you to attend the opening ceremony of our new building at No. 27 Bristol Street, Fort, Colombo, on the 30th May, 1965, the 28th Day of Muharram 1385, Sunday afternoon, $4: 15 \mathrm{pm}$.

Honourable Prime Minister Mr. Dudley Senanayake will unveil the commemoration block. The President of the centre, Sir Razik Fareed will open the building.

Specimen of Arabu-Tamil writing (Computer encoded) ----- 2

The following are the few couplets of an Arabu-Tamil poem written by Syed Mohammed Imam al-Aroos(1816-1898 A.D):

1.

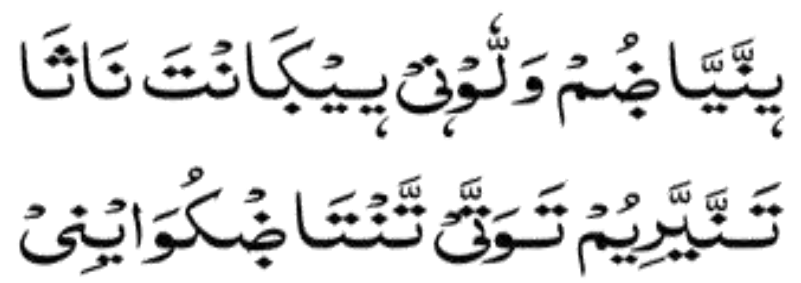

(Transliteration of the above couplet in Tamil) என்னை ஆளும் வல்லோனே ஏகாந்த நாதா தன்னை அறியும் தவத்தை தந்தாள்குவாய் நீ 


\section{(Translation of the above couplet in English)}

O Almighty Who rules over me! O my Master who is the only Lord!

Bless me with the boon of realizing the insignificance of myself.

2.

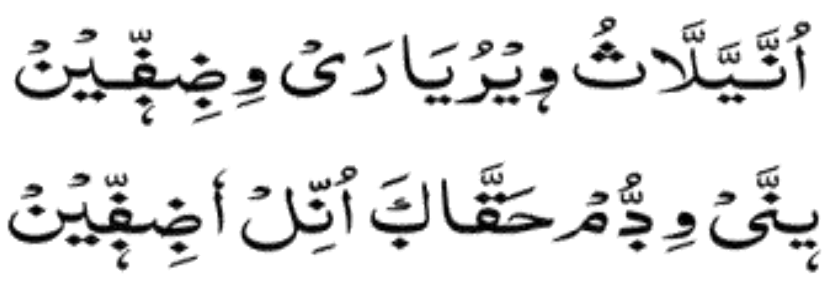

(Transliteration of the above couplet in Tamil)

உன்னை அல்லாது வேறு யாரை விளிப்பேன் என்னை விட்டும் ஹக்காக உன்னில் ஒளிப்பேன்

(Translation of the above couplet in English)

Whom can I beseech except Thee!

I will [leave my wretched self and] annihilate in Thee!

\section{Status and recognition of Arabu-Tamil}

The UNO has accepted Arabu-Tamil as one of the approved languages of the world. "The UNESCO COURIER" published by the UNESCO, in August - September 1981, has published an article on the life of the Holy prophet, written by Mohammed Yaasin. ${ }^{12}$

\section{Development of Arabu-Tamil Language and Literature}

This new language became the mother tongue of the Muslim community. It is developed as the medium for their day-to-day affairs including business, property dealings, correspondence, and all other social transaction.

Literature produced in prose and poetry in this unique language covers a vast field of subjects like
1. Architecture
2. Astronomy
3. Creed
4. Biography
5. Commentary on Quran
6. Dictionary
7. Elegy
8. Fiction
10. History
11. Horticulture
9. Islamic Jurisprudence
12. Logic 

13. Medicine
14. Moral science
15. Satire
16. Sexology
17. Sports
18. Mysticism etc.

This language was developed during the early medieval ages. Its literature is mostly of religious character. Tamil scholars, through their works, contributed immensely to the development of this language and literature. In the words of Edgard Thurston, Arabu-Tamil is a literature which was developed for the purpose of the education of Labbai $^{13}$ and Maraikayar ${ }^{14}$ children. The Koran and other books were published in this language.

The origin of this literature may be traced to Kayalpattnam ${ }^{15}$, Melapalayam and other inimportant Labbai Towns in the Tirunelveli District $^{16}$. The orthographic practices adopted by the many authors who wrote in Arabu-Tamil were not all alike. The consequent variations are noticeable in their writings. In linguistic term the language is composed of more than one set of grammars and vocabularies that a speaker may switch back and forth, depending on the situation. As a written language it employs an invented orthography for a creolized, or mixed, system of speech patterns. The structure and principles of its development are possibly related to similar systems known for other similar Islamic speech and writing systems such as Swahili language of Tanzania of East Africa, Jaawi language of Malaysia and languages of other countries namely Turkey, Uzbek, Berber, Somali and Malay.

Four hundred years ago, a particular procedure and a new literary style was adopted in Arabu-Tamil writing. This new literary style was adopted by Hafiz Amir Wali Appa, a saint of Kayalpattinam. He is considered to be the first person who re-introduced Arabu-Tamil after the Portuguese devastation in around $1600 .{ }^{17}$ His tomb is available in the weaver's street, in Kayalpattinam. The style of Hafiz Amir Wali Appa perfected the Arabu-Tamil to a large extent. Method of its writing and speaking, though existed even before Hafiz Amir Wali Appa, was not systematic. It was purely a colloquial one. Amir Wali was the first man to systematize it. Its literature flourished even in Burma and the Far Eastern countries where the Tamil Muslims lived. A book in Arabu-Tamil by the name "Haqiqatul Insan" was printed in Rangoon, in 1882. The book contains 174 pages. Three more books were written and published from Akyab, Mohmein of Burma (Presently Myanmar). According to A.M.A. Azeez of Sri Lanka, the Leiden university in Holland has several Arabu-Tamil books written 
by Muslims of Tamil Nadu and Sri Lanka. There are Arabu-Tamil schools functioning on an elaborate scale in Malaysia, Singapore, Burma and Pakistan (Karachi) even today. According to Aziz, out of 15,000 books kept in the Indian Office Library in London, nearly 60 are in Arabu-Tamil; famous among them are Nondi Nadagam (1872) and Sira Nadagam (1878).

The First commentary of Holy Quran by Tamil scholar was written in Arabu-Tamil language. It was written by Shaykh Mustafa Wali (18361887). The five commentaries have been published in Arabu-Tamil language are as follows:

a. Fathhul Kareem by Nooh Lebbai, son of Abdul Qader of Kayalpattinam. This was printed in two volumes in Bombay (Mumbai) in 1299AH|1881AD.

b. Fathhul Raheem: Nooh Lebbai also wrote another commentary called Fathhul Raheem, which is shorter than his own Fathhul Kareem. It exists in six parts of 288 pages, printed at Bombay (Mumbai) in $1304 \mathrm{AH}$.

c. Futhuhathur Rahmania by Habeeb Mohammed Alim, published in the year $1296 \mathrm{AH} \mid 1875 \mathrm{AD}$. This commentary was printed in Bombay (Mumbai) in Husayni press.

d. Fathhur Rahman fi Tarjamati Tafsiril Quran. This Arabu-Tamil commentary was written by Shaykh Mustafa Wali (12521305AH /1836-1887AD) of Srilanka.

e. Rahmatul Mannan. Sulaiman bin Mohammed of Sri Lanka has written this commentary. This was printed in Colombo in 1315AH / 1897AD.

Sheik Mustafa Alim of Peruvalai, Sri Lanka, is considered the great translator in Arabu-Tamil. He translated the following works from Arabic into Arabu-Tamil language:

a. Fawaariqul Hidayaa- it is a translated work of Sahlud Dowlah

b. Sermons of Ibn nabata

c. Safeenatun Najaath

d. Fawaaidul Aqaaid of Ihyaa Uloomuddin. 
Sam Shihabuddin Wali (1634-1709) was a great poet in Arabu-Tamil. He was the first person to compose poetic work in Arabu-Tamil. ${ }^{18}$ The following poets were considered great masters in Arabu-Tamil :

a. Sheik Mustafa Alim of Peruvalai, Sri Lanka

b. Sheik Mohammed Lebbai bin Ahmed Kaswatta Alim of Sri Lanka

c. Sheik Jamaluddin

d. Sheik Mohiyuddin Mena Hajiar of Kottaru, Tamil Nadu

e. Sheik Madina Sahib

f. Sheik Syed Mohammed Mapillay Lebbai Alim

g. Sheik Sam Shihabuddin of Kayalpattanam, Tamil Nadu.

h. Sheik Abdul Qadir Naina Lebbai Pulavar Nayagam

i. Sheik Mohammed Sadaqatullah Jin Kaatib.

Arabu-Tamil Periodicals were as follows ${ }^{19}$

a. Allamat Lankapuri -A Journal which was published in Colombo from 1869.

b. Ajaibul Akhbar - A Weekly which was published in Madras(Chennai) in 1870's.

c. Muslim Nesan- A Newspaper which was published in Colombo from 21-12-1882.

d. Kashfurran an Qalbil Jan which was published in Colombo from 1889.

\section{Contribution of Arabu-Tamil}

The religious revival of the Muslims was made possible because of this voluminous literature in Arabu-Tamil produced by the Muslim Savants after the devastating cultural onslaught by the Portuguese in this area in $16^{\text {th }}$ century. This onslaughts took place in 1528 and in 1537. Literature produced in this language covered the fields of Jurisprudence, Sufism, Law, Medicine, and Poetry. Arabu-Tamil was also used as a bridge language for Tamil Muslims to learn Arabic. Many authentic Hadith manuscripts are produced in this language. Most of the Fiqh books, particularly those of Imaam Shaafi and Imaam Abu Hanifa are found in 
Arabu-Tamil. There was even a translation of the Bible into ArabuTamil in 1926. The Arabu-Tamil therefore contributed immensely to the education of Muslims in South India and Sri Lanka.

Compositions of devotional songs used in certain Islamic ceremonies were originally written in Arabu-Tamil by their authors. Thalai Fathiha is in Arabu-Tamil Script, seeking the favour of Allah, through the spiritual assistance of Hazrath Fatima Zahra and the prophets and saints. He has given a fine and enviable description of the life in paradise and has also given a fearful picture of the despicable life in the hell. It was read in South India, Ceylon (Sri Lanka) and the far East with great fervor and spirit at the end of the devotional meetings. ${ }^{20}$ Thalai Fathiha - a well known Arabu-Tamil song is devotionally sung by women in ceremonies.

There are thousands of Arabu-Tamil books on different subjects. Names of the various books on different subjects with their authors are systematically organized, in the Madras Archives Catalogues. These catalogues are maintained from 1890. Periods of the catalogues are as follows: 1890-1901; 1901-1910; 1911-1915 and 1921-25. According to the catalogues, there are more than 3,000 Arabu-Tamil books. The most important book is Arabu-Tamil Dictionary in four bulky volumes written by Hakkim Mohammed Abdullah Sahib. This literature helped the Tamil Muslims to know about their religion and their culture. And in a way this literature also made the Arabic literature simpler. ${ }^{21}$

\section{Decline of Arabu-Tamil Language}

Arabu-Tamil (Arwi) language originated in $8^{\text {th }}$ century and flourished during the medieval ages saw its decline from the beginning of the twentieth century. In the Globalizational Era, no step was taken to arrest this decline of this language which had the south Asia based cultural and historical background. Today Arabu-Tamil is known to be a matter of least scholarly interest in some parts of Tamil Nadu state of India and Sri Lanka. This literature did not receive much attention in the later part of the $20^{\text {th }}$ century. Other languages such as English replaced it in many contexts. One most important reason for its decline is the lack of competitive printing facilities. Printing of Arabu-Tamil books is a very complicated and tedious process. Therefore, people preferred printing their religious books in Tamil rather than in Arabu-Tamil language. During the early 20th century the adoption of Urdu as the dominant Islamic school curriculum, which is ethnocentrically unconcerned with 
Arabu-Tamil which has a different South Asia based cultural and historical background, is also another reason for its decline.

\section{Conclusion}

In the system of modern education, Muslim children failed to learn Arabu-Tamil language, as it was out of the school curriculum. In the system of religious educational institutions, the north Indian syllabus known as Silsilatun Nizamiyyah was adopted by the Madrasahs in the beginning of the twentieth century in Tamil Nadu. Arabu-Tamil as a language and subject did not find a place in that syllabus.

The decline of Arabu-Tamil language is a great loss to the Tamil Muslim community as this was their religious language. It had safeguarded the interest of this community. It was fondly and reverently nurtured by noble savants. Therefore, its necessity must be felt by the present Tamil Muslim community. Its revival and renaissance is badly needed. The following steps are suggested to arrest its decline and revitalize its literature for the cultural benefit of particularly the Tamil Muslim Community:

a. All the Arabu-Tamil language books available should be collected and re-printed. Unpublished manuscripts also should be collected and printed

b. Arabu-Tamil language should be made compulsory in all the Madrasahs of Tamilnadu and Sri Lanka.

c. Arabu-Tamil books must be taught to children attending schools as a part of school curriculum.

d. Arabu-Tamil community must be encouraged to use it in their daily affairs.

e. Periodicals and magazines in this language should be re-introduced.

With these measures taken we may witness the period of renaissance in Arabu-Tamil language and literature in the years to come. 


\section{References}

1 on line available at: http://en.wikipedia.org/wiki/Arwi_language

2 ABDUR RAHIM,M.R.M (1976), Islamia Kalai Kalanjiam (Tamil/Islamic Encyclopedia),Madras, ( Chennai), Universal Publishers, pp. 415-416.

3 JOHN SAMUEL, DR.G. (2010), Tamil as a classical Language, Chennai,pp:271.

$4 \quad$ Islamic Studies, Vol.34:,No.;4,pp:407,408

5 Islamic Studies, Vol.34:,No.;4,pp: 409

6 ABDUR RAHMAN, H. (April 1985), Origin and Development of ArabuTamil in Tamil Nadu,Chennai,pp.23.

7 SHUYAB ALIM, DR. TAYKA (1993), Arabic, Arwi and Persian in Sarandib and Tamil Nadu, Chennai,pp.91.

8 JOHN SAMUEL, DR.G (2010), Tamil as a classical Language, Chennai,pp:273.

9 ABDUR RAHMAN, H. (April1985) Origin and Development of ArabuTamil in Tamil Nadu, Chennai, pp.25.

10 JOHN SAMUEL, DR. G. (2010), Tamil as a classical Language, Chennai, pp:275.

11 Muslim Tamils of Srilanka were referred by the pejorative term Sonaga or Sonagar

12 SHUYAB ALIM, DR. TAYKA (1993), Arabic, Arwi and Persian in Sarandib and Tamil Nadu, Chennai, pp.90.

13 Labbai is a distinctive Islamic community from Southern India. A Trading Community spread throughout the southern states of Tamil Nadu, Kerala, Andhra Pradesh and Karnataka, Labbais follow either the Hanafi or Shafi'i school of Fiqh.

14 Maraikayar is a distinctive Tamil- and Malayalam-speaking Muslim people of the states of Kerala and Tamil Nadu in India. Some of the places abundant with Maricars are Kayalpatnam (which is thought to be the ancestral hometown of the early Maricars), Kilakarai, Adirampattinam, Muthupet, Nagore, Nagapattinam, Karaikal and various other coastal towns.Traditionally, the Maricars engaged in mercantile commerce. They generally dealt with gems, precious stones, jewels, timber and the textile trade.

15 Kayalpatnam is a town in the Tuticorin (formerly of Tirunelveli) district of Tamil Nadu, India. Kayalpatnam is an ancient historical city. It lies in the southeast part of Indian peninsula, on the shore off the Bay of Bengal. It is 
a Muslim dominant town. It is situated about 400 miles from Madras (Chennai) and about 100 miles from Trivandrum and Madurai. Kayalpatnam was an important trade emporium even before the advent of Islam.

16 JOHN SAMUEL, DR. G. (2010), Tamil as a classical Language, Chennai, pp:271.

17 RIZWANUR RAHMAN (Chief Editor), Thaqafatul Hind, Vol.57:, No.;2, pp:189.

18 JOHN SAMUEL, DR. G. (2010), Tamil as a classical Language, Chennai, pp:276, 277.

19 SHUYAB ALIM, DR. TAYKA (1993), Arabic, Arwi and Persian in Sarandib and Tamil Nadu, Chennai, pp.103.

20 MOHAMMAD YOUSUF KOKAN (1974), Arabic and Persian in Carnatic (1710-1960), Hafiza House, Madras, pp:522

21 JOHN SAMUEL, DR.G. (2010), Tamil as a classical Language, Chennai, pp:277.

\section{Works cited}

a. ABDUR RAHIM, M. R. M (1976), Islamia Kalai Kalanjiam (Tamil/Islamic Encyclopedia), Madras, (Chennai), Universal Publishers.

b. ABDUR RAHMAN, H. (April 1985), Origin and Development of ArabuTamil in Tamil Nadu,. (M. Phil Dissertation Submitted to the University of Madras).

c. Islamic Studies,(PAKISTAN) Vol.34:,No.;4,pp:407,408. An English Article Entitled Spoken Tamil dialect of the Muslims of Sri Lanka: Language as Identity classifier published by M M M MAHAROOF.

d. JOHN SAMUEL, DR. G. (Chief Editor/2010), Tamil as a classical Language,(A collection of Papers presented in the first International symposium on Tamil As a Classical language)Chennai. An English Article Entitled Tamil Arabic Relationship presented by Dr.AHAMED ZUBAIR K.M.A.pp:271-277.

e. MEERAN PILLAI, M.M. (December 1990), Aayvu Kovai, \{A collection of Papers presented in the fifth International Islamic Tamil Conference A Tamil Research article Entitled Arabu-Tamilin Thotram-Oor Aayvu /(The Origin of Arabu -Tamil: A Study) presented in the Fifth 
International Islamic Tamil Conference Held at Kilakarai in 1990, pp:335-347.

f. MOHAMMAD YOUSUF KOKAN (1974), Arabic and Persian in Carnatic (1710-1960), Hafiza House, Madras.

g. RIZWANUR RAHMAN (Chief Editor), Thaqafatul Hind(New Delhi, India),Vol.57:, No.;2. An Arabic Article Entitled al-Lisan al-Arwi published by Dr. AHAMED ZUBAIR K.M.A. pp:188-204. ISSN No: 0970-3713.

h. SHAHUL HAMEED, K.S.M. ( October-November 2003, Manarul Huda (Islamic Tamil Monthly), Chennai (A Tamil Article entitled ArabuTamilin Thevai, Thotram, Theivu/The Necessity, Origin and the Decline of Arabu-Tamil, pp:63-67.)

i. SHUYAB ALIM, DR. TAYKA (1993), Arabic, Arwi and Persian in Sarandib and Tamil Nadu, Imaamul Aroos Trust, Chennai. 